\title{
Investigation of a quantum mechanical detector model for moving, spread-out particles
}

\author{
G C Hegerfeldt ${ }^{1}$, J T Neumann ${ }^{1}$ and L S Schulman ${ }^{2}$ \\ ${ }^{1}$ Institut für Theoretische Physik, Universität Göttingen, Friedrich-Hund-Platz 1, \\ 37077 Göttingen, Germany \\ 2 Physics Department, Clarkson University, Potsdam, NY 13699-5820, USA \\ E-mail: hegerf@theorie.physik.uni-goettingen.de,neumann@theorie.physik.uni-goettingen.de \\ and schulman@clarkson.edu
}

Received 7 September 2006, in final form 10 October 2006

Published 1 November 2006

Online at stacks.iop.org/JPhysA/39/14447

\begin{abstract}
We investigate a fully quantum mechanical spin model for the detection of a moving particle. This model, developed in earlier work, is based on a collection of spins at fixed locations and in a metastable state, with the particle locally enhancing the coupling of the spins to an environment of bosons. The appearance of bosons from particular spins signals the presence of the particle at the spin location, and the first boson indicates its arrival. The original model used discrete boson modes. Here we treat the continuum limit, under the assumption of the Markov property, and calculate the arrival-time distribution for a particle to reach a specific region.
\end{abstract}

PACS numbers: $03.65 . \mathrm{Xp}, 03.65 . \mathrm{Ta}, 05.50 .+\mathrm{q}$

\section{Introduction}

Until recently, in time-of-flight measurements for particles or atoms the quantum nature of the centre-of-mass motion usually played no role since the particles or atoms were very fast. However, the advance of cooling techniques has made it possible to create ultracold gases in a trap and produce very slow atoms, e.g., by opening the trap. For these low velocities the quantum nature of the centre-of-mass motion of an atom can have noticeable quantum effects, as the remarkable experiments of Szriftgiser et al have shown [1]. In the simplest quantum mechanical formulation of a time-of-flight measurement one would create a particle at $t=0$ with a localized but extended wavefunction and then ask for the arrival time of the particle at some distant point. Repeating this one would get an arrival-time distribution that would depend on the particle's wavefunction. Similarly one might ask for passage or transit times through a region. Such questions, and more generally the role of time in quantum mechanics, have attracted much interest in recent years [2,3]. 
But how should one measure the arrival time of a particle or atom at some particular point and what should the resulting distribution look like? Allcock [4] made an ad hoc model of an arrival-time measurement using an imaginary step potential, which leads to an 'absorption' of the wave packet; he then identified the absorption rate with the arrival-time distribution. In general, this distribution will not be normalized since part of the wave packet will be reflected from the imaginary step potential rather than being absorbed. Also, part of the wave packet may penetrate the step to some depth before being absorbed, thus causing a detection 'delay.' As Allcock noticed, decreasing one effect will typically enlarge the other.

Kijowski proposed physically motivated axioms from which he derived an 'ideal' arrivaltime distribution for a free quantum particle coming from one direction [5]. The resulting distribution agrees with an 'approximate distribution' proposed heuristically by Allcock. This distribution has been related [6] to the arrival-time operator of Aharonov and Bohm [7] (for more on the latter see [8] and references therein). No measurement procedure for the distribution was proposed, and its status, properties and generalizations are still being critically discussed in the literature; see e.g. [9-11].

Halliwell [12] employed a detection model based on a single spin coupled to a boson bath, a greatly simplified version of a general quantum mechanical detector model that was proposed in [13] and elaborated in [3, 14]. Working in one space dimension and using Bloch equations he arrived at a Schrödinger equation with an imaginary potential, thus giving a basis for Allcock's approach.

An operational and realistic laser-based approach to the arrival-time problem was investigated in [15-21]. This approach proposes to measure the arrival time by means of laser-induced fluorescence [15]. The idea is to consider a two-level atom with centre-of-mass motion, to illuminate some region of space with a laser, and to take the detection time of the first fluorescence photon as the arrival time of the atom at the (sharp) onset of the laser. In this approach one has to deal with the typical problems of delay due to the time needed for pumping and decay of the excited state. There is also reflection without detection when the atom is reflected from the laser beam in the ground state without emitting a photon. Yet, interesting results could be derived. In the limit of a weak laser, there is almost no reflection but a strong delay due to the weak pumping to the upper energy level of the two-level system; dealing with this delay by means of a deconvolution, one recovers the flux at the position of the onset of the laser from the first-photon distribution [15]. On the other hand, in the limit of strong pumping, reflection becomes dominant and the first-photon distribution is clearly not normalized; normalizing it via the operator normalization method of Brunetti and Fredenhagen, which preserves the bilinear structure of the distribution [22], one recovers Kijowski's arrivaltime distribution from the first-photon distribution [16]. In this way, Kijowski's axiomatic distribution can be related to a particular measuring process. Further, in a certain limit it is possible to derive a closed one-channel equation for the ground state governing the first-photon distribution [17]. This equation contains in general a complex potential which becomes purely imaginary for zero laser detuning. In this way the fluorescence model makes a connection to Allcock's ad hoc ansatz of an imaginary potential.

In the fluorescence model there is a back-reaction of the measurement on the centre-ofmass motion of the atom, and this might cause deviations from an ideal distribution. It thus seems a good idea to use a measurement procedure that does not interact directly with the particle through its internal degrees of freedom, but rather to regard the particle only as a catalyst for a transition in a detector or its associated environment.

Just such a detection model was developed in [3, 13, 14]. The model consists of a three-dimensional array of $D$ spins (the 'detector') with ferromagnetic interaction. In the presence of a homogeneous magnetic field, and for sufficiently low temperature, all spins 
are aligned with the field. Reversing the magnetic field suddenly, such that the spins cannot follow the reversal, one can produce a metastable state of this compound spin system. The spins are weakly coupled to a bath of bosons. There is a particle to be detected and its effect on the collection of spins is to strongly enhance the spin-boson coupling when the particle's wavefunction overlaps that of a detector spin. Thus when the particle is close to a spin this spin flips much faster by virtue of the increased coupling to the bath. By means of the ferromagnetic interaction, this in turn triggers the subsequent spontaneous flipping of all spins even in the absence of the particle. In this way, the single-spin flip is amplified to a macroscopic event and the associated bosons can be measured. The details of the amplification process and the probability of false detection due to spontaneous spin flips were considered in $[3,13,14]$. The motion of the particle, whose presence induces the first spin flip, was treated classically in the calculations. In the following, we will concentrate on the full quantum description of this first spin flip and the quantum mechanical aspects of the particle's motion, and comment only briefly on processes internal to the detector.

In this paper we investigate this detector model in the limit of continuous boson modes, under the condition that the spin-boson interaction satisfies the Markov property (see (43)), and use it to determine the arrival-time distribution of a spatially spread-out particle. It turns out that one is again led to a Schrödinger equation with an imaginary potential and the corresponding arrival-time distribution is similar to that of the fluorescence model. In this detector model there is also a back-reaction on the particle of interest. In order to eliminate this back-reaction we discuss the idea of decreasing the spin-bath coupling while simultaneously increasing the number of spins. It is shown that even in the limit when the spin-bath coupling goes to zero and the number of spins to infinity, there remains a back-reaction.

The plan of the paper is as follows. In section 2 the detector model is reviewed, and in section 3 the arrival-time distribution obtained from a simplified version of the model is calculated by means of standard quantum mechanics. Another approach to calculate the arrival-time distribution is presented in section 4 and compared to the straightforward calculation. The advantage of this second approach is that is easily extended to the full model, the corresponding calculations shown in the appendix, and that it allows to some extent for an analytical treatment of the arrival-time problem. In section 5 we discuss the relation of the present detection scheme to the fluorescence model. Section 6 deals with the limit of zero coupling and an infinity of spins, and remarks on possible schemes for the optimization of the model and on its application to passage-time measurements.

\section{The detector model}

The detector model of $[3,13,14]$ is based on the following Hamiltonian. The excited state of the $j$ th spin is denoted by $|\uparrow\rangle_{j}$ and its ground state by $|\downarrow\rangle_{j}$. Define

$$
\hat{\sigma}_{z}^{(j)} \equiv|\uparrow\rangle_{j j}\langle\uparrow|-| \downarrow\rangle_{j j}\langle\downarrow| \text {. }
$$

The Hamiltonian for the detector alone is given by

$$
H_{\mathrm{det}}=\frac{1}{2} \sum_{j} \hbar \omega_{0}^{(j)} \hat{\sigma}_{z}^{(j)}-\frac{1}{2} \sum_{j<k} \hbar \omega_{J}^{(j k)} \hat{\sigma}_{z}^{(j)} \otimes \hat{\sigma}_{z}^{(k)},
$$

where $\hbar \omega_{0}^{(j)}$ is the energy difference between ground state and excited state of the $j$ th spin, and $\hbar \omega_{J}^{(j k)} \geqslant 0$ is the coupling energy between the spins $j$ and $k$.

In addition there is a bath of bosons (e.g., phonons or photons) with the free Hamiltonian

$$
H_{\text {bath }}=\sum_{\ell} \hbar \omega_{\ell} \hat{a}_{\ell}^{\dagger} \hat{a}_{\ell}
$$


where $\hat{a}_{\ell}$ is the annihilation operator for a boson with wave vector $\ell$. Later a continuum limit will be taken. In general, the spins will be coupled to the bath, and there is the possibility of spontaneous spin flips due to

$$
H_{\text {spon }}=\sum_{j, \ell} \hbar\left(\gamma_{\ell}^{(j)} \mathrm{e}^{\mathrm{i} f_{\ell}^{(j)}} \hat{a}_{\ell}^{\dagger} \hat{\sigma}_{-}^{(j)}+\text { h.c. }\right) \text {, }
$$

where

$$
\hat{\sigma}_{-}^{(j)} \equiv|\downarrow\rangle_{j j}\left\langle\uparrow\left|, \quad \hat{\sigma}_{+}^{(j)} \equiv\left(\hat{\sigma}_{-}^{(j)}\right)^{\dagger}=\right| \uparrow\right\rangle_{j j}\langle\downarrow|,
$$

and the coupling constants $\gamma_{\ell}^{(j)}$ and the phases $f_{\ell}^{(j)}$ depend on the particular realization of the detector and the bath.

The coupling between the $j$ th spin and the bath is assumed to be strongly enhanced when the particle is close to this spin. Let the $j$ th spin be located in a spatial region $\mathcal{G}_{j}$. The enhancement is taken to be proportional to a sensitivity function $\chi^{(j)}(\mathbf{x})$ which vanishes outside $\mathcal{G}_{j}$, e.g. the characteristic function which is 1 on $\mathcal{G}_{j}$ and zero outside. The additional coupling depending on the particle's position is thus

$$
H_{\text {coup }}=\sum_{j} \chi^{(j)}(\hat{\mathbf{x}}) \sum_{\ell} \hbar\left(g_{\ell}^{(j)} \mathrm{e}^{\mathrm{i} f_{\ell}^{(j)}} \hat{a}_{\ell}^{\dagger} \hat{\sigma}_{-}^{(j)}+\text { h.c. }\right),
$$

with $\left|g_{\ell}^{(j)}\right|^{2} \gg\left|\gamma_{\ell}^{(j)}\right|^{2}$.

The full Hamiltonian is

$$
H=H_{\text {part }}+H_{\text {det }}+H_{\text {bath }}+H_{\text {spon }}+H_{\text {coup }},
$$

where $H_{\text {part }}$ is the free Hamiltonian of the particle,

$$
H_{\text {part }}=\hat{\mathbf{p}}^{2} / 2 m \text {. }
$$

Note that the 'excitation number', i.e., the sum of the number of bosons and the number of up-spins, is a conserved quantity. The detection process now starts with the bath in its ground state $|0\rangle$ (no bosons present) and all $D$ spins in the excited state $\left|\uparrow_{1} \ldots \uparrow_{D}\right\rangle$. As a consequence of the excitation number conservation, it is sufficient to measure the state $|0\rangle$ of the bath in order to check whether or not any spin has flipped. For $\hbar \omega_{0}^{(j)}$ only slightly above the energetic threshold set by the ferromagnetic spin-spin coupling, and $\gamma_{\ell}^{(j)}$ sufficiently small, the probability of a spontaneous spin flip ('false positive') is very small $[3,13,14]$. But when the particle is close to the $j$ th spin, the excited state $|\uparrow\rangle_{j}$ decays much more quickly, due to the enhanced coupling, ' $g_{\ell}^{(j)}$, , of the spin to the bath. Then, the ferromagnetic force experienced by its neighbours is strongly reduced, and thus these spins can flip rather quickly even in the absence of the particle by means of the $\gamma_{\ell}^{(j)}$; by a kind of 'domino effect', the whole array of spins will eventually flip, amplifying the first spin flip to a macroscopic event $[3,13,14]$.

\section{The direct approach in the one-spin case}

\subsection{A simplified model}

We first consider a simplified model consisting of a particle in one dimension and only one spin. This simplification is reasonable if the radius of the region $\mathcal{G}_{j}$ is smaller than the distance between spins. (Our assumption of locality of the interaction though is a bit stronger than this however, since in section 3.2, for calculational convenience we will extend the region $\mathcal{G}_{j}$ to a half-line, i.e., $\chi(x) \rightarrow \Theta(x)$.) The vectors $\mathbf{x}$ and $\ell$ are replaced by $x$ and $\ell$. Also, we will temporarily neglect $H_{\text {spon }}$ in view of assumption $\left|\gamma_{\ell}^{(j)}\right|^{2} \ll\left|g_{\ell}^{(j)}\right|^{2}$, and accordingly the possibility of spontaneous spin flips. 
The free Hamiltonian for the particle motion in one dimension is

$$
H_{\text {part }}^{1 \mathrm{~d}}=\hat{p}^{2} / 2 m \text {, }
$$

and the free detector Hamiltonian with only one spin simplifies to

$$
H_{\mathrm{det}}^{1}=\frac{1}{2} \hbar \omega_{0} \hat{\sigma}_{z} \text {. }
$$

The free bath Hamiltonian is given by

$$
H_{\mathrm{bath}}^{1 \mathrm{~d}}=\sum_{\ell} \hbar \omega_{\ell} \hat{a}_{\ell}^{\dagger} \hat{a}_{\ell}
$$

Furthermore, let the spin be located in the interval $\mathcal{I}_{d} \equiv[0, d]$ so that

$$
H_{\text {coup }}^{1,1 \mathrm{~d}}=\chi_{I_{d}}(\hat{x}) \sum_{\ell} \hbar\left(g_{\ell} \mathrm{e}^{\mathrm{i} f_{\ell}} \hat{a}_{\ell}^{\dagger} \hat{\sigma}_{-}+\text {h.c. }\right)
$$

where the sensitivity function $\chi_{\mathcal{I}_{d}}(x)$ vanishes outside $\mathcal{I}_{d}$. The full Hamiltonian of the simplified model is then given by

$$
H^{1,1 \mathrm{~d}}=H_{\text {part }}^{1 \mathrm{~d}}+H_{\mathrm{det}}^{1}+H_{\text {bath }}^{1 \mathrm{~d}}+H_{\text {coup }}^{1,1 \mathrm{~d}} .
$$

This simplified model allows for a direct investigation by means of standard quantum mechanics.

\subsection{Energy eigenstates}

To get a first idea of how the present detector model works for an arrival-time measurement, we simplify the model in this section a little further by assuming the detector to be semi-infinite, extended over the whole positive axis, and take momentarily

$$
\chi_{\mathcal{I}_{d}}(x)=\Theta(x),
$$

where $\Theta$ is Heaviside's step function. Also, we assume for the phases in the coupling Hamiltonian $f_{\ell} \equiv 0$ throughout this section. The stationary Schrödinger equation with energy eigenvalue $E_{k}$ for a plane wave coming in from the left, initially no bosons present, and the spin in state $|\uparrow\rangle$, can be solved piecewise in position space. For $x<0$, the solution simply reads

$$
\boldsymbol{\Phi}_{k}^{<}(x)=\sqrt{\frac{1}{2 \pi}}\left(\left[\mathrm{e}^{\mathrm{i} k x}+R_{0}(k) \mathrm{e}^{-\mathrm{i} k x}\right]|\uparrow 0\rangle+\sum_{\ell} R_{\ell}(k) \mathrm{e}^{-\mathrm{i} k_{\ell}(k) x}\left|\downarrow 1_{\ell}\right\rangle\right),
$$

where the wave numbers $k, k_{\ell}(k)$ are fixed by

$$
\frac{\hbar^{2} k^{2}}{2 m}+\frac{\hbar \omega_{0}}{2}=E_{k}=\frac{\hbar^{2} k_{\ell}(k)^{2}}{2 m}-\frac{\hbar \omega_{0}}{2}+\hbar \omega_{\ell},
$$

and where $|\uparrow 0\rangle \equiv|\uparrow\rangle|0\rangle,\left|\downarrow 1_{\ell}\right\rangle \equiv|\downarrow\rangle\left|1_{\ell}\right\rangle$. Note that there is the possibility that the particle is reflected from the detector. It may either be reflected after it has been detected and a boson of mode $\ell$ has been created, the coefficient for this event being $R_{\ell}(k)$, or it may even be reflected without being detected, the coefficient being $R_{0}(k)$. The latter will lead to a non-normalized arrival-time distribution. Since this no-detection probability is in general momentum dependent the momentum distribution of the actually detected part of the wave packet must be expected to differ from that of the originally prepared wave packet, hence leading to deviations of the 'measured' arrival-time distribution from corresponding 'ideal' quantities.

For $x>0$, the operator $H^{1,1 \mathrm{~d}}-\hat{p}^{2} / 2 m$ is independent of $x$, because $\chi_{\mathcal{I}_{d}}(x)=\Theta(x)$ has been assumed, and it commutes with $\hat{p}^{2} / 2 m$. The eigenvalues of $H^{1,1 \mathrm{~d}}-\hat{p}^{2} / 2 m$ are real and 
denoted by $\hbar \Omega_{\mu} / 2$. The corresponding eigenvectors are superpositions of $|\uparrow 0\rangle$ and $\left|\downarrow 1_{\ell}\right\rangle$ and denoted by $|\boldsymbol{\mu}\rangle$ so that

$$
\left(H^{1,1 \mathrm{~d}}-\hat{p}^{2} / 2 m\right)|\boldsymbol{\mu}\rangle=\frac{\hbar \Omega_{\mu}}{2}|\boldsymbol{\mu}\rangle .
$$

To obtain an eigenvector of $H^{1,1 \mathrm{~d}}$ on $x>0$ for the eigenvalue $E_{k}$, one has to choose an eigenfunction $\mathrm{e}^{\mathrm{i} q_{\mu}(k) x}$ of $\hat{p}^{2} / 2 m$ such that

$$
E_{k}=\left(\hbar q_{\mu}(k)\right)^{2} / 2 m+\hbar \Omega_{\mu} / 2 \text {. }
$$

From (15) one has

$$
q_{\mu}(k)=\sqrt{k^{2}+\frac{m}{\hbar}\left(\omega_{0}-\Omega_{\mu}\right)} .
$$

Note that $q_{\mu}(k)$ is imaginary if $\Omega_{\mu}>\omega_{0}$ and

$$
k^{2}<\frac{m}{\hbar}\left(\Omega_{\mu}-\omega_{0}\right)
$$

leading to exponential decay. Otherwise $q_{\mu}(k)$ is real. The solution of the stationary Schrödinger equation for $x>0$ belonging to the eigenvalue $E_{k}$ can then be written as

$$
\boldsymbol{\Phi}_{k}^{>}(x)=\sqrt{\frac{1}{2 \pi}} \sum_{\mu} \alpha_{\mu}(k) \mathrm{e}^{\mathrm{i} q_{\mu}(k) x}|\boldsymbol{\mu}\rangle .
$$

The coefficients $\alpha_{\mu}(k), R_{0}(k), R_{\ell}(k)$ are obtained from the usual matching condition, i.e. both

$$
\boldsymbol{\Phi}_{k}(x):=\left\{\begin{array}{lll}
\boldsymbol{\Phi}_{k}^{<}(x) & \text { if } \quad x<0 \\
\boldsymbol{\Phi}_{k}^{>}(x) & \text { if } \quad x \geqslant 0
\end{array}\right.
$$

and its first derivative have to be continuous at $x=0$. The eigenvectors $|\boldsymbol{\mu}\rangle$ can be determined numerically.

\subsection{Detection of a wave packet}

The probability of finding the detector spin in state $|\downarrow\rangle$ (and hence the bath in some boson state $\left.\left|1_{\ell}\right\rangle\right)$ at time $t$ is given by integration over the modulus square of the respective component of $\left|\Psi_{t}\right\rangle$

$$
\begin{aligned}
P_{1}^{\mathrm{disc}}(t) & =\sum_{\ell} \int_{-\infty}^{\infty} \mathrm{d} x\left|\left\langle x \downarrow 1_{\ell} \mid \Psi_{t}\right\rangle\right|^{2} \\
& =1-\int_{-\infty}^{\infty} \mathrm{d} x\left|\left\langle x \uparrow 0 \mid \Psi_{t}\right\rangle\right|^{2} \equiv 1-P_{0}^{\mathrm{disc}}(t),
\end{aligned}
$$

where the superscript 'disc' distinguishes the discrete model from the continuum limit discussed in the next section. As long as no recurrences occur, i.e., no transitions $\left|\downarrow 1_{\ell}\right\rangle \mapsto|\uparrow 0\rangle$, one can regard

$$
w_{1}^{\text {disc }}(t)=\frac{\mathrm{d}}{\mathrm{d} t} P_{1}^{\mathrm{disc}}(t)=-\frac{\mathrm{d}}{\mathrm{d} t} P_{0}^{\mathrm{disc}}(t)
$$

as the probability density for a spin flip (i.e., for a detection) at time $t$.

As an example we consider a maximal boson frequency $\omega_{\mathrm{M}}$ and

$$
\omega_{\ell}=\omega_{\mathrm{M}} n / N \quad n=1, \ldots, N \quad g_{\ell}=-\mathrm{i} G \sqrt{\omega_{\ell} / N} .
$$

As particle we consider a cesium atom, prepared in the remote past far away from the detector such that the corresponding free packet (i.e., in the absence of the detector) at $t=0$ would 


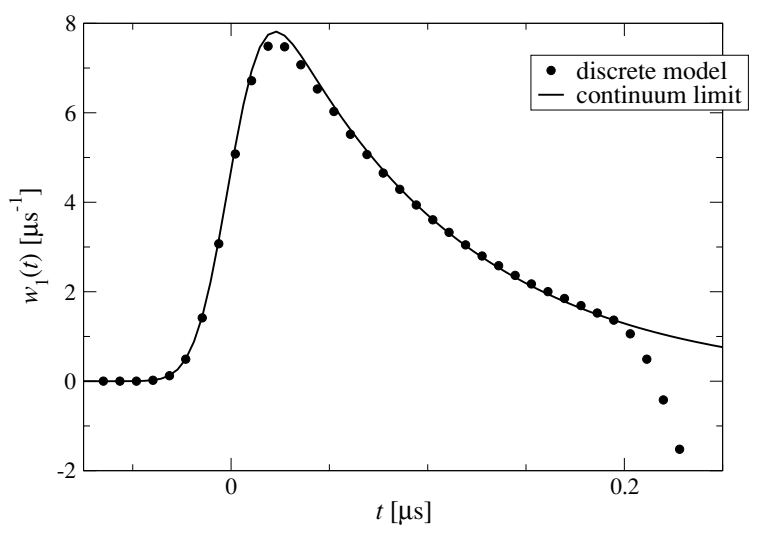

Figure 1. Dots: spin-flip probability density $w_{1}^{\text {disc }}(t)$ for an incoming Gaussian wave packet of (25) and (26) with $\Delta p=20 \mu \mathrm{m}^{-1} \hbar$ and $v_{0}=1.79 \mathrm{~m} \mathrm{~s}^{-1} ; \omega_{0}=2.39 \times 10^{8} \mathrm{~s}^{-1}, \omega_{\mathrm{M}}=4.6 \omega_{0}, G=$ $2.782 \times 10^{3} \mathrm{~s}^{-1 / 2}, N=40$. Solid line: $w_{1}(t)$ from (52) for the corresponding continuum limit. Up to the time of recurrences, $\left|\downarrow 1_{\ell}\right\rangle \mapsto|\uparrow 0\rangle$ (due to the discrete nature of the bath), the discrete and continuum probability densities are in good agreement.

be a Gaussian minimal uncertainty packet around $x=0$ with $\Delta p$ and average velocity $v_{0}$. Decomposing this into the eigenstates of $H$, the wave packet at time $t$ is

$$
\left\langle x \mid \Psi_{t}\right\rangle=\int_{-\infty}^{\infty} \mathrm{d} k \widetilde{\psi}(k) \Phi_{k}(x) \mathrm{e}^{-\mathrm{i} E_{k} t / \hbar}
$$

with

$$
\widetilde{\psi}(k)=\left(\frac{\hbar}{\Delta p \sqrt{2 \pi}}\right)^{1 / 2} \exp \left(-\frac{\hbar^{2}}{4(\Delta p)^{2}}\left(k-m v_{0} / \hbar\right)^{2}\right) .
$$

A numerical illustration of $w_{1}^{\text {disc }}(t)$ for $N=40$ is given in figure 1 (dots). The numerical calculation is time consuming, while in the continuous case with the quantum jump approach it is much faster (see the next section).

\section{Continuum limit and quantum jump approach}

\subsection{Basic ideas}

In this section the detector model and its application to arrival times will be investigated in a continuum limit by means of the quantum jump approach [23]. This approach uses continuous bath modes as a limit, so that there are no recurrences as in the discrete case. It is easily generalized to multiple spins and it is more accessible to analytic treatment. The bath modes are eliminated, but in contrast to Bloch equations one can work with a (conditional or effective) Hamiltonian and has reduced dimensions. It is based on watching for the first appearance of a boson. To do this one would have to observe the bath continuously. Since in standard quantum mechanics with the simple von Neumann measurement theory this would lead to difficulties associated with the quantum Zeno effect [24-26], the quantum jump approach circumvents this by temporally coarse-grained observations and a coarse-grained time scale. In the present situation, it reads as follows. Instead of continuous observation, one considers repeated instantaneous measurements, separated by a time $\Delta t$. For a Markovian system with correlation time $\tau_{\mathrm{c}}$, one takes $\Delta t \gg \tau_{\mathrm{c}}$ to avoid the quantum Zeno effect, but $\Delta t$ 
much shorter than the lifetime of the excited state $\left|\uparrow_{1} \ldots \uparrow_{D}\right\rangle$ in order to obtain a good time resolution. Typical numbers for quantum optical models are $\Delta t \simeq 10^{-13} \mathrm{~s} \ldots 10^{-10} \mathrm{~s}$. To find no boson until $t=n \Delta t$, no boson must have been found in the first $n$ measurements. The probability for this to happen will now be calculated. The detector interval $\mathcal{I}_{d}$ can now be finite or semi-infinite.

Let the complete system (bath, detector, and particle) at $t_{0}=0$ be prepared in the state

$$
\left|\Psi_{0}\right\rangle=|0\rangle\left|\uparrow_{1} \ldots \uparrow_{D}\right\rangle\left|\psi_{0}\right\rangle,
$$

where $\left|\psi_{0}\right\rangle$ denotes the spatial wavefunction of the particle. If no boson is found at the first measurement then, by the von Neumann-Lüders reduction rule [27, 28], the state (up to normalization) right after the measurement is given by projecting with $|0\rangle\langle 0|$,

$$
\left|\Psi_{\text {cond }}^{\Delta t}\right\rangle \equiv|0\rangle\langle 0|U(\Delta t, 0)| 0\rangle\left|\uparrow_{1} \ldots \uparrow_{D}\right\rangle\left|\psi_{0}\right\rangle,
$$

where $U\left(t, t^{\prime}\right)$ denotes the time-evolution operator of the complete system. The probability, $P_{0}(\Delta t)$, for no detection is the norm squared of the vector in (28), i.e.

$$
P_{0}(\Delta t)=\||0\rangle\langle 0|U(\Delta t, 0)| 0\rangle\left|\uparrow_{1} \ldots \uparrow_{D}\right\rangle\left|\psi_{0}\right\rangle \|^{2} .
$$

The state then evolves with $U(2 \Delta t, \Delta t)$ until the next measurement, and so on. The state after the $n$th consecutive no-boson measurement, $\left|\Psi_{\text {cond }}^{n \Delta t}\right\rangle$, is, up to normalization,

$$
\begin{aligned}
\left|\Psi_{\text {cond }}^{n \Delta t}\right\rangle= & |0\rangle\langle 0|U(n \Delta t,[n-1] \Delta t)| 0\rangle \cdots \\
& \cdots\langle 0|U(\Delta t, 0)| 0\rangle\left|\uparrow_{1} \ldots \uparrow_{D}\right\rangle\left|\psi_{0}\right\rangle .
\end{aligned}
$$

The probability, $P_{0}(n \Delta t)$, of finding the bath in the state $|0\rangle$ in all of the first $n$ measurements is given by its norm squared,

$$
P_{0}(n \Delta t)=\left\langle\Psi_{\text {cond }}^{n \Delta t} \mid \Psi_{\text {cond }}^{n \Delta t}\right\rangle .
$$

Note that $\langle 0|U(v \Delta t,[v-1] \Delta t)| 0\rangle$ is an operator in the particle-detector Hilbert space which does not rotate $\left|\uparrow_{1} \ldots \uparrow_{D}\right\rangle$, by excitation number conservation mentioned after (8). Thus one can write

$$
\left|\Psi_{\text {cond }}^{n \Delta t}\right\rangle=\left|\Psi_{\text {cond }}^{t}\right\rangle \equiv|0\rangle\left|\uparrow_{1} \ldots \uparrow_{D}\right\rangle\left|\psi_{\text {cond }}^{t}\right\rangle,
$$

where $t=n \Delta t$, and hence

$$
P_{0}(t) \equiv\left\langle\Psi_{\text {cond }}^{n \Delta t} \mid \Psi_{\text {cond }}^{n \Delta t}\right\rangle=\left\langle\psi_{\text {cond }}^{t} \mid \psi_{\text {cond }}^{t}\right\rangle
$$

is the probability that no transition $|0\rangle \longrightarrow\left|1_{\ell}\right\rangle$, i.e. that no detection occurs until the time $t$. The probability for the first detection to occur at next measurement is just given by

$$
P_{0}(t)-P_{0}(t+\Delta t) \equiv w_{1}(t) \Delta t .
$$

The crucial point now is to calculate the 'conditional time evolution' of $\left|\psi_{\text {cond }}^{t}\right\rangle$, i.e., the time evolution 'under the condition that no detection occurs', and for this one has to evaluate $\langle 0|U(v \Delta t,[v-1] \Delta t)| 0\rangle\left|\uparrow_{1} \ldots \uparrow_{D}\right\rangle$.

\subsection{A simplified model}

For greater clarity, the evaluation of $\langle 0|U(v \Delta t,[v-1] \Delta t)| 0\rangle\left|\uparrow_{1} \ldots \uparrow_{D}\right\rangle$ will first be done for the simplified model introduced at the beginning of section 3 , while the generalization to the full model is referred to the appendix. 
We use the interaction picture w.r.t. $H_{0}^{1,1 \mathrm{~d}}=H^{1,1 \mathrm{~d}}-H_{\text {coup }}^{1,1 \mathrm{~d}}$ and $U_{I}\left(t, t^{\prime}\right)=$ $\mathrm{e}^{\frac{\mathrm{i}}{\hbar} H_{0}^{1,1 \mathrm{ld}} t} U\left(t, t^{\prime}\right) \mathrm{e}^{-\frac{\mathrm{i}}{\hbar} H_{0}^{1,1 \mathrm{~d}} t^{\prime}}$. Using (12) with still discrete, but possibly infinitely many, modes a simple calculation gives in second order perturbation theory

$\left\langle 0\left|U_{I}(v \Delta t,[v-1] \Delta t)\right| 0\right\rangle|\uparrow\rangle=|\uparrow\rangle$

$$
\times\left(\mathbb{1}-\int_{[v-1] \Delta t}^{v \Delta t} \mathrm{~d} t_{1} \int_{[v-1] \Delta t}^{t_{1}} \mathrm{~d} t_{2} \sum_{\ell} \chi_{\mathcal{I}_{d}}\left(\hat{x}\left(t_{1}\right)\right) \chi_{\mathcal{I}_{d}}\left(\hat{x}\left(t_{2}\right)\right)\left|g_{\ell}\right|^{2} \mathrm{e}^{\mathrm{i}\left(\omega_{0}-\omega_{\ell}\right)\left(t_{1}-t_{2}\right)}\right),
$$

where $\hat{x}(t)=\hat{x}+\hat{p} t / m$ is the time evolution of the operator $\hat{x}$ in the Heisenberg picture of the free particle. The phases in the coupling terms have cancelled; even if one would assume these phases to be dependent on the particle's position, $f_{\ell}(x)$, this would be the case to very good approximation since $\Delta t$ is very small and thus $\hat{x}\left(t_{1}\right) \approx \hat{x}\left(t_{2}\right)$ [29]. Consequently one obtains

$\left\langle 0\left|U_{I}(v \Delta t,[v-1] \Delta t)\right| 0\right\rangle|\uparrow\rangle=|\uparrow\rangle$

$$
\times\left(\mathbb{1}-\int_{[v-1] \Delta t}^{v \Delta t} \mathrm{~d} t_{1} \int_{[v-1] \Delta t}^{t_{1}} \mathrm{~d} t_{2} \chi_{I_{d}}\left(\hat{x}\left(t_{1}\right)\right) \chi_{I_{d}}\left(\hat{x}\left(t_{2}\right)\right) \cdot \kappa\left(t_{1}-t_{2}\right)\right)
$$

with the correlation function

$$
\kappa(\tau) \equiv \sum_{\ell}\left|g_{\ell}\right|^{2} \mathrm{e}^{-\mathrm{i}\left(\omega_{\ell}-\omega_{0}\right) \tau}
$$

To have irreversible decay we go to the continuum limit as follows. At first the bath modes are indexed by 'wave numbers'

$$
\ell=2 \pi n / L_{\text {bath }}, \quad n=1,2, \ldots
$$

and $\omega_{\ell}$ is chosen as

$$
\omega_{\ell}=c\left(\omega_{\ell}\right) \ell
$$

so that

$$
\Delta \omega=\frac{c(\omega)^{2}}{c(\omega)-\omega c^{\prime}(\omega)} \Delta \ell=\frac{c(\omega)^{2}}{c(\omega)-\omega c^{\prime}(\omega)} \cdot \frac{2 \pi}{L_{\text {bath }}} .
$$

The coupling constants are taken to be of the form

$$
g_{\ell}=\left(\Gamma\left(\omega_{\ell}\right)+\mathcal{O}\left(L_{\text {bath }}^{-1}\right)\right) \cdot \sqrt{\frac{\omega_{\ell}}{L_{\text {bath }}}},
$$

where $\Gamma\left(\omega_{\ell}\right)$ does not depend on $L_{\text {bath. }}$. Then one obtains in the continuum limit by (40)

$$
\kappa(\tau)=\frac{1}{2 \pi} \int_{0}^{\infty} \mathrm{d} \omega \frac{c(\omega)-\omega c^{\prime}(\omega)}{c(\omega)^{2}} \omega|\Gamma(\omega)|^{2} \mathrm{e}^{-\mathrm{i}\left(\omega-\omega_{0}\right) \tau} .
$$

We assume $\Gamma(\omega)$ to be of such a form that the Markov property holds, i.e.

$$
\kappa(\tau) \approx 0 \quad \text { if } \quad \tau>\tau_{\mathrm{c}}
$$

for some small correlation time $\tau_{\mathrm{c}}$. This is the case, e.g., for $\Gamma(\omega) \equiv \Gamma$ as in quantum optics. In the double integral of (36) then only times with $t_{1}-t_{2} \leqslant \tau_{\mathrm{c}}$ contribute, and if $\tau_{\mathrm{c}}$ is small enough one can write

$$
\chi_{I_{d}}\left(\hat{x}\left(t_{1}\right)\right) \chi_{I_{d}}\left(\hat{x}\left(t_{2}\right)\right) \approx \chi_{I_{d}}\left(\hat{x}\left(t_{1}\right)\right)^{2} .
$$

The double integral then becomes

$$
\int_{0}^{\Delta t} \mathrm{~d} t^{\prime} \chi_{I_{d}}\left(\hat{x}\left(t^{\prime}+(v-1) \Delta t\right)\right)^{2} \int_{0}^{t^{\prime}} \mathrm{d} \tau \kappa(\tau)
$$


With $\Delta t \gg \tau_{\mathrm{c}}$ the second integral can be extended to infinity, by the Markov property. Putting

$$
\begin{aligned}
& A \equiv 2 \operatorname{Re} \int_{0}^{\infty} \mathrm{d} \tau \kappa(\tau)=\omega_{0} \frac{c\left(\omega_{0}\right)-\omega_{0} c^{\prime}\left(\omega_{0}\right)}{c\left(\omega_{0}\right)^{2}} \cdot\left|\Gamma\left(\omega_{0}\right)\right|^{2} \\
& \delta_{\text {shift }} \equiv 2 \operatorname{Im} \int_{0}^{\infty} \mathrm{d} \tau \kappa(\tau)
\end{aligned}
$$

one obtains

$$
\begin{gathered}
\left\langle 0\left|U_{I}(v \Delta t,[v-1] \Delta t)\right| 0\right\rangle|\uparrow\rangle=|\uparrow\rangle\left(\mathbb{1}-\frac{1}{2}\left(A+\mathrm{i} \delta_{\text {shift }}\right) \int_{[v-1] \Delta t}^{v \Delta t} \mathrm{~d} t_{1} \chi_{I_{d}}\left(\hat{x}\left(t_{1}\right)\right)^{2}\right) \\
=|\uparrow\rangle \exp \left(-\frac{1}{2}\left(A+\mathrm{i} \delta_{\text {shift }}\right) \int_{[v-1] \Delta t}^{v \Delta t} \mathrm{~d} t_{1} \chi_{I_{d}}\left(\hat{x}\left(t_{1}\right)\right)^{2}\right),
\end{gathered}
$$

up to higher orders in $\Delta t$. Note that $A$ is a decay rate of the upper spin level; in quantum optics $A$ and $\delta_{\text {shift }}$ correspond to the Einstein coefficient and to a line shift. Going back to the Schrödinger picture one then obtains by (30)

$$
\left|\psi_{\text {cond }}^{t}\right\rangle=\mathrm{e}^{-\frac{\mathrm{i}}{\hbar} H_{\text {cond }}\left(t-t_{0}\right)}\left|\psi_{0}\right\rangle
$$

with the 'conditional Hamiltonian'

$$
H_{\text {cond }} \equiv \frac{\hat{p}^{2}}{2 m}+\frac{\hbar}{2}\left(\delta_{\text {shift }}-\mathrm{i} A\right) \chi_{\mathcal{I}_{d}}(\hat{x})^{2} .
$$

Note that this result is independent of the particular choice of $\Delta t$ as long as $\Delta t$ satisfies the above requirements. As a consequence, on a coarse-grained time scale in which $\Delta t$ is small, $t$ can be regarded as continuous and $\left|\psi_{\text {cond }}^{t}\right\rangle$ obeys a Schrödinger equation with a complex potential,

$$
\mathrm{i} \hbar \frac{\partial}{\partial t}\left|\psi_{\text {cond }}^{t}\right\rangle=\left(\frac{\hat{p}^{2}}{2 m}+\frac{\hbar}{2}\left(\delta_{\text {shift }}-\mathrm{i} A\right) \chi_{\mathcal{I}_{d}}(\hat{x})^{2}\right)\left|\psi_{\text {cond }}^{t}\right\rangle
$$

In this continuous, coarse-grained, time scale, (34) yields for the probability density, $w_{1}(t)$, for the first detection

$$
w_{1}(t)=-\frac{\mathrm{d} P_{0}(t)}{\mathrm{d} t}
$$

and from (33) and (48) one easily finds

$$
\begin{aligned}
w_{1}(t) & =\frac{\mathrm{i}}{\hbar}\left\langle\psi_{\text {cond }}^{t}\left|H_{\text {cond }}-H_{\text {cond }}^{\dagger}\right| \psi_{\text {cond }}^{t}\right\rangle \\
& =A \int_{0}^{d} \mathrm{~d} x \chi_{\mathcal{I}_{d}}(x)^{2}\left|\left\langle x \mid \psi_{\text {cond }}^{t}\right\rangle\right|^{2}
\end{aligned}
$$

If $\chi_{I_{d}}(\hat{x})$ is the characteristic function of the interval $[0, d]$ this is just the decay rate of the excited state of the detector multiplied by the probability that the particle is inside the detector but not yet detected-a very physical result. 


\subsection{An example}

As an example we consider the continuum limit of the discrete model of (24). In this case one has, with $\omega_{\mathrm{M}}$ being the maximal frequency,

$$
\begin{aligned}
& c(\omega) \equiv c_{0} \\
& \omega_{\ell}=\omega_{\mathrm{M}} n / N \equiv c_{0} 2 \pi n / L_{\text {bath }}, \quad n=1, \ldots, N \\
& L_{\text {bath }} \equiv 2 \pi c_{0} N / \omega_{\mathrm{M}} \\
& g_{\ell}=-\mathrm{i} G \sqrt{\omega_{\ell} / N} \equiv-\mathrm{i} G \sqrt{2 \pi c_{0} / \omega_{\mathrm{M}}} \sqrt{\frac{\omega_{\ell}}{L_{\text {bath }}}} \\
& \Gamma(\omega)=\left\{\begin{array}{cl}
-\mathrm{i} G \sqrt{2 \pi c_{0} / \omega_{\mathrm{M}}} \equiv \Gamma & \text { if } \omega \leqslant \omega_{\mathrm{M}} \\
0 & \text { else. }
\end{array}\right.
\end{aligned}
$$

In the continuum limit, $N$ or $L_{\text {bath }} \rightarrow \infty$, one obtains in the case $\omega_{\mathrm{M}}>\omega_{0}$

$$
\begin{aligned}
& \kappa(\tau)=\frac{|G|^{2}}{\omega_{\mathrm{M}}} \cdot \frac{\left(1+\mathrm{i} \omega_{\mathrm{M}} \tau\right) \mathrm{e}^{-\mathrm{i}\left(\omega_{\mathrm{M}}-\omega_{0}\right) \tau}-\mathrm{e}^{\mathrm{i} \omega_{0} \tau}}{\tau^{2}} \\
& A=2 \pi|G|^{2} \frac{\omega_{0}}{\omega_{\mathrm{M}}} \\
& \delta_{\text {shift }}=2|G|^{2}\left(\frac{\omega_{0}}{\omega_{\mathrm{M}}} \ln \left[\frac{\omega_{0}}{\omega_{\mathrm{M}}-\omega_{0}}\right]-1\right)
\end{aligned}
$$

and $\tau_{\mathrm{c}}$ is of the order of $\omega_{0}^{-1}$. In the integral for $w_{1}(t)$ in (52) one has $\chi_{I_{d}}(x)=\Theta(x)$. The resulting $w_{1}(t)$ is plotted in figure 1 for the same wavefunction and parameters as for $w_{1}^{\text {disc }}(t)$ in that figure. Both distributions are in good agreement up to the occurrence of recurrences $\left|\downarrow 1_{\ell}\right\rangle \mapsto|\uparrow 0\rangle$ in the discrete case.

The agreement is also seen for other values of $\Delta p$. If $\Delta x$ is the width of the wave packet in position space and $v_{0}$ is its average velocity, then the width of the probability density for detection is at least of the order of $\Delta x / v_{0}$ since it takes some time for the wave packet to enter the detection region. (Further broadening of the detection density arises from the width of the delay of the first spin flip once the particle is inside the detector.) Consequently, wave packets with small $\Delta p$ and thus large $\Delta x$ yield rather broad detection densities. On the other hand, as soon as a significant part of the wavefunction overlaps the detector, in the discrete case the time scale of the recurrences is essentially determined by the properties of the detector and the bath and by their coupling. In the case of wave packets with small $\Delta p$ long recurrence times are needed to obtain a good resolution of the typically broad detection densities. This requires a large number of bath modes in the discrete case. We further note that for more complicated incident wave packets as, e.g., the coherent superposition of several Gaussian wave packets with different mean velocities, the probability density exhibits a more complicated structure due to the self-interference of the wavefunction.

\subsection{The general case}

A procedure analogous to (35)-(52) can be applied to the three-dimensional model with several spins, as explained in the appendix. The bosons are allowed to have a direction $\mathbf{e}$ which varies over the unit sphere. In a continuum limit $\left|\psi_{\text {cond }}^{t}\right\rangle$ obeys a Schrödinger equation with a complex potential

$$
\mathrm{i} \hbar \frac{\partial}{\partial t}\left|\psi_{\text {cond }}^{t}\right\rangle=H_{\text {cond }}\left|\psi_{\text {cond }}^{t}\right\rangle
$$


where

$$
H_{\text {cond }}=\frac{\hat{\mathbf{p}}^{2}}{2 m}+\frac{\hbar}{2}\left\{\delta_{\text {shift }}(\hat{\mathbf{x}})-\mathrm{i} A(\hat{\mathbf{x}})\right\}
$$

$A(\mathbf{x})$ and $\delta_{\text {shift }}(\mathbf{x})$ are given in (A.8) and (A.9). The probability density for the first detection is again similar to (52),

$$
\begin{aligned}
w_{1}(t) & =\frac{\mathrm{i}}{\hbar}\left\langle\psi_{\text {cond }}^{t}\left|H_{\text {cond }}-H_{\text {cond }}^{\dagger}\right| \psi_{\text {cond }}^{t}\right\rangle \\
& =\int \mathrm{d}^{3} x A(\mathbf{x})\left|\left\langle\mathbf{x} \mid \psi_{\text {cond }}^{t}\right\rangle\right|^{2},
\end{aligned}
$$

which is an average of the position-dependent decay rate of the detector, weighted with the probability density for the particle to be at position $\mathbf{x}$ and yet undetected.

\section{Relation of the present detector model to the fluorescence model}

In the quantum optical fluorescence model [15-21] for arrival times one considers a two-level atom with ground state $|1\rangle \equiv\left(\begin{array}{l}1 \\ 0\end{array}\right)$ and excited state $|2\rangle \equiv\left(\begin{array}{l}0 \\ 1\end{array}\right)$, which enters a laser illuminated region. In the one-dimensional case one obtains a conditional Hamiltonian of the form

$$
H_{\text {cond }}^{\mathrm{fl}}=\frac{\hat{p}^{2}}{2 m}+\frac{\hbar}{2}\left(\begin{array}{cc}
0 & \Omega(\hat{x}) \\
\Omega(\hat{x}) & -\mathrm{i} \gamma-2 \Delta
\end{array}\right),
$$

where $\Omega(x)$ is the (position dependent) Rabi frequency of the laser, $\Delta$ is the detuning (possibly also position dependent) and $\gamma$ is the decay constant of the excited level. Note that in contrast to the present model this is a two-channel Hamiltonian. The reason for this is that the ground state of the quantized photon field is not related to a specific internal state of the atom due to the driving by the (classical) laser. In the limit

$$
\frac{\hbar|2 \Delta+\mathrm{i} \gamma|}{2} \gg \frac{\hbar}{2} \Omega, E
$$

where $E$ denotes the kinetic energy of the incident particle, the corresponding conditional Schrödinger equation reduces to a one-channel equation for the ground state amplitude with the complex potential

$$
V(x)=\frac{\hbar \Delta \Omega(x)^{2}-\mathrm{i} \hbar \gamma \Omega(x)^{2} / 2}{4 \Delta^{2}+\gamma^{2}},
$$

and the excited state can be neglected in this limit [17]. Physically, condition (59) means that the excited state decays very rapidly compared to the time scales of the pumping and the centre-of-mass motion. Thus, the first fluorescence photon is emitted, i.e., the particle is detected, when and where the excitation takes place. For $\Delta \equiv 0$ (laser in resonance) $V$ is a purely imaginary potential, similar as for the detector model outlined above; only the physical interpretation of the height of this imaginary potential differs. In other words, the one-channel limit of the fluorescence model coincides with the full quantum mechanical model from section 2 when considering the conditional interaction for the particle until the first detection. In this way, the fully quantum mechanical detector model of section 2 not only justifies the fluorescence model for quantum arrival times, at least in the limit of (59), but one can conversely immediately carry over the results of the fluorescence model to the detector model. The investigation of the fluorescence model has shown that the essential features such as reflection and delay [15], and main results such as, e.g., linking Kijowski's arrival-time distribution to a particular measuring process [16], can be obtained from the full two-channel model as well as from its one-channel limit. Hence these results immediately carry over to the 
present detector model. Also, the derivation of a complex potential model for particle detection from two different physical models, viz the fluorescence model and the present detector model, indicates the importance of the complex potentials and of Kijowski's arrival-time distribution, which in turn can be derived from the complex potentials approach. This connection is interesting since it can illuminate the physical background of otherwise heuristically introduced complex potentials. Differences, however, arise for example in applications to passage times since the reset state after a detection is not the same in the two models [32].

\section{Discussion and extensions}

The model investigated in this paper has three ingredients, viz a particle in whose spatial properties one is interested, a 'detector' based on spins, and a bath of bosons, originally in the ground state. There is neither a direct measurement on the particle of interest nor on the detector but only on the bath, which is checked for bosons. In this way one can hope to keep the disturbance of the particle by the measurement to a minimum. However, as in the fluorescence model and also seen in [12] for a simplified spin model, also the present full model yields a description by a complex potential and thus shows the typical unwanted features: there is a detection delay, due to the finite spin decay or flip rate, and there is also necessarily the possibility of reflection of the particle by the detector without the detection of a boson, due to the increased bath-detector coupling caused by the particle's wavefunction inside the detector. This reflection without boson detection causes a non-detection of the particle so that the probability density $w_{1}(t)$ in (57) for the first detection is not normalized. A similar effect arises from the transmission of the particle without boson detection.

In order to reduce the detection delay one may be tempted to increase the spin-bath coupling, which mirrors the particle's wavefunction inside the detector. As a by-product this would also decrease transmission without detection. However, the increase of this spatially dependent coupling means an increase of the absorbing potential $-\mathrm{i} \hbar A(\mathbf{x}) / 2$, and this will also increase the reflection without boson detection, so much so that in the limit of infinite coupling everything is reflected while nothing is detected. The same phenomenon occurs in the fluorescence model [15] and is a typical feature of complex potentials, as already noted by Allcock [4].

One can also try to reduce the influence of the spin-bath system on the particle and thus the latter's disturbance by decreasing the spin-bath coupling at a space point and simultaneously increasing the number of spins located there. This seems natural because it is the flip of a single spin which gives rise to the detection, and with a larger number of spins this can compensate for the weaker coupling. To investigate this quantitatively we consider $N$ spins, later to be taken to $\infty$, in the same volume $V$ and $\chi^{(j)}(\mathbf{x}) \equiv \chi_{V}(\mathbf{x})$ for all $j$. The coupling constants are taken in the form

$$
g_{\ell}^{(j)} \equiv g_{\ell}=\frac{\Gamma\left(\omega_{\ell}, \mathbf{e}_{\ell}\right)+\mathcal{O}\left(L_{\text {bath }}^{-1}\right)}{\sqrt{N}} \cdot \sqrt{\frac{\omega_{\ell}}{L_{\text {bath }}^{3}}}
$$

and similarly for $\gamma_{\ell}^{(j)}$. Further, the ferromagnetic force experienced by the individual spin is assumed not to grow with increasing $N$ such as for nearest-neighbour interaction. Then (A.8) becomes

$$
\begin{aligned}
A(\mathbf{x}) & =\sum_{j=1}^{N}\left(\tilde{\omega}_{0}\right)^{3}\left[\frac{c\left(\tilde{\omega}_{0}\right)-\tilde{\omega}_{0} c^{\prime}\left(\tilde{\omega}_{0}\right)}{c\left(\tilde{\omega}_{0}\right)^{4}}\right] \int \frac{\mathrm{d} \Omega_{\mathbf{e}}}{(2 \pi)^{2}} \frac{\left|\Gamma\left(\tilde{\omega}_{0}, \mathbf{e}\right)\right|^{2} \chi_{V}(\mathbf{x})^{2}+\left|\Gamma_{\text {spon }}\left(\tilde{\omega}_{0}, \mathbf{e}\right)\right|^{2}}{N} \\
& =\left(\tilde{\omega}_{0}\right)^{3}\left[\frac{c\left(\tilde{\omega}_{0}\right)-\tilde{\omega}_{0} c^{\prime}\left(\tilde{\omega}_{0}\right)}{c\left(\tilde{\omega}_{0}\right)^{4}}\right] \int \frac{\mathrm{d} \Omega_{\mathbf{e}}}{(2 \pi)^{2}}\left(\left|\Gamma\left(\tilde{\omega}_{0}, \mathbf{e}\right)\right|^{2} \chi_{V}(\mathbf{x})^{2}+\left|\Gamma_{\text {spon }}\left(\tilde{\omega}_{0}, \mathbf{e}\right)\right|^{2}\right)
\end{aligned}
$$


which is just the decay rate for a single spin in $V$, with resonance frequency $\tilde{\omega}_{0}$ and the coupling as for $N=1$. A similar result holds for $\delta_{\text {shift }}(\mathbf{x})$, defined in (A.9).

Thus, simply increasing the number of spins $N$ and scaling the coupling constants with $\sqrt{1 / N}$ leaves $A$ and $\delta_{\text {shift }}$ invariant and thus does not change the dynamics until the first detection (spin flip), and in particular does not help to avoid reflection of undetected particles. Any other scaling power of $N$, however, would not lead to a reasonable detector model in the limit $N \rightarrow \infty$ since then either $A$ and $\delta_{\text {shift }}$ would go to zero or to $\infty$. Similar results also hold for the quantum optical fluorescence model. It is interesting to note that, although it is the flip of one single spin which triggers the detection, it is the totality of all spins located in $V$ which determines the conditional time evolution.

It has been shown in the context of complex potentials, however, that one can deal with the delay/transmission-versus-reflection problem by dropping the restriction to rectangular potentials $[30,31]$. In fact, it is possible to absorb nearly the complete wave packet in a very short spatial interval; given a wave packet with a specific energy range, an appropriate imaginary potential can be constructed by means of inverse scattering techniques. We stress that there is no such a thing as the optimal imaginary potential for all wave packets but the construction of the optimized potential requires a priori information about energy range of the wave packet under consideration.

The present detector model is applicable not only to arrival-time measurements, but also to more involved tasks such as a measurement of passage times. A detailed analysis including numerical examples will appear elsewhere [32]. It turns out that a too weak spinbath coupling yields a broad passage-time distribution due to the slow response of the detector to the presence of the particle. A too strong spin-bath coupling, on the other hand, also yields a broad passage-time distribution due to the strong distortion of the wave packet during the measurement process. This is a quantum effect. There is, however, an intermediate range for $A(\mathbf{x})$ yielding rather narrow passage-time distributions. Indeed, a rough estimate in [32] shows that for an optimal choice of incident wave packet and decay rate $A(\mathbf{x})$ the precision of the measurement can be expected to behave like $E^{-3 / 4}$, where $E$ is the energy of the incident particle. For low velocities, this means some improvement as compared to the results of models coupling the particle continuously or semi-continuously to a clock, where one has $E^{-1}$ behaviour [33,34]. Thus, it appears that the latter $E^{-1}$ behaviour of the precision is not due to a fundamental limitation related to a kind of time-energy uncertainty relation.

\section{Summary}

We have investigated the continuum limit of a fully quantum mechanical spin model for the detection of a moving particle when the spin-boson interaction satisfies the Markov property. In an example with a single spin and 40 boson modes it was shown numerically that the continuum limit gave a good approximation to the discrete model up to times of revivals. We have derived analytical expressions for the arrival-time distribution. The conditional Schrödinger equation governing the particle's time evolution before the detection has the same form as the one-channel limit of the fluorescence model, which is based on the use of a laser-illuminated region. The quantum spin detector model provides an easier way to obtain this one-channel equation, since no additional assumptions or limits are needed.

\section{Acknowledgment}

This work was supported in part by NSF Grant PHY 0555313. 


\section{Appendix. The quantum jump approach for several spins}

The continuum limit and quantum jump approach for the full model in section 2 is quite similar until (35). In second order perturbation theory w.r.t. $H_{\text {coup }}+H_{\text {spon }}$ one obtains

$$
\begin{aligned}
& \left\langle 0\left|U_{I}(v \Delta t,[v-1] \Delta t)\right| 0\right\rangle\left|\uparrow_{1} \ldots \uparrow_{D}\right\rangle=\left|\uparrow_{1} \ldots \uparrow_{D}\right\rangle\left(\mathbb{1}-\sum_{j, \ell} \int_{[v-1] \Delta t}^{v \Delta t} \mathrm{~d} t_{1} \int_{[v-1] \Delta t}^{t_{1}} \mathrm{~d} t_{2}\right. \\
& \left.\times \mathrm{e}^{\mathrm{i}\left(\widetilde{\omega}_{0}^{(j)}-\omega_{\ell}\right)\left(t_{1}-t_{2}\right)} \overline{\left(\chi^{(j)}\left(\hat{\mathbf{x}}\left(t_{1}\right)\right) g_{\ell}^{(j)}+\gamma_{\ell}^{(j)}\right)} \cdot\left(\chi^{(j)}\left(\hat{\mathbf{x}}\left(t_{2}\right)\right) g_{\ell}^{(j)}+\gamma_{\ell}^{(j)}\right)\right),
\end{aligned}
$$

where

$$
\widetilde{\omega}_{0}^{(j)} \equiv \omega_{0}^{(j)}-\left(\sum_{k=1}^{j-1} \omega_{J}^{(k j)}+\sum_{k=j+1}^{D} \omega_{J}^{(j k)}\right)
$$

are modified resonance frequencies arising from the ferromagnetic spin-spin coupling. The phases $f_{\ell}^{(j)}$ have cancelled similar to the one-spin case since only products of the form $\hat{a}_{\ell} \hat{\sigma}_{+}^{(j)} \hat{a}_{\ell}^{\dagger} \hat{\sigma}_{-}^{(j)}$ contribute to the second order, and consequently the contributions from different spins do not mix.

Similar to (42) one can define correlation functions $\kappa_{\bar{g} g}^{(j)}, \kappa_{\bar{g} \gamma}^{(j)}, \kappa_{\bar{\gamma} g}^{(j)}$ and $\kappa_{\bar{\gamma} \gamma}^{(j)}$ in an obvious way. Before the continuum limit the bath modes are indexed by the wave vectors

$$
\ell=\frac{2 \pi}{L_{\text {bath }}}\left(\begin{array}{l}
n_{1} \\
n_{2} \\
n_{3}
\end{array}\right), \quad n_{i}=1,2, \ldots
$$

In analogy to (41), the coupling constants are taken in the form

$$
\begin{aligned}
& g_{\ell}^{(j)}=\left(\Gamma^{(j)}\left(\omega_{\ell}, \mathbf{e}_{\ell}\right)+\mathcal{O}\left(L_{\text {bath }}^{-1}\right)\right) \cdot \sqrt{\frac{\omega_{\ell}}{L_{\text {bath }}^{3}}} \\
& \gamma_{\ell}^{(j)}=\left(\Gamma_{\text {spon }}^{(j)}\left(\omega_{\ell}, \mathbf{e}_{\ell}\right)+\mathcal{O}\left(L_{\text {bath }}^{-1}\right)\right) \cdot \sqrt{\frac{\omega_{\ell}}{L_{\text {bath }}^{3}}}
\end{aligned}
$$

with $\omega_{\ell}=c\left(\omega_{\ell}\right) \ell, \mathbf{e}_{\ell}=\ell / \ell$, and

$$
\left|\Gamma^{(j)}\left(\omega_{\ell}, \mathbf{e}_{\ell}\right)\right|^{2} \gg\left|\Gamma_{\text {spon }}^{(j)}\left(\omega_{\ell}, \mathbf{e}_{\ell}\right)\right|^{2} .
$$

Again the Markov property is assumed to hold for the correlation functions in the continuum limit. The procedure is then analogous to the single-spin case, and one obtains (48) with the conditional Hamiltonian

$$
H_{\text {cond }}=\frac{\hat{\mathbf{p}}^{2}}{2 m}+\frac{\hbar}{2}\left\{\delta_{\text {shift }}(\hat{\mathbf{x}})-\mathrm{i} A(\hat{\mathbf{x}})\right\}
$$

where $A(\mathbf{x})$ is given in analogy to (46) by

$$
\begin{aligned}
A(\mathbf{x})= & 2 \operatorname{Re} \sum_{j} \int_{0}^{\infty} \mathrm{d} \tau\left\{\kappa_{\bar{g} g}^{(j)}(\tau) \chi^{(j)}(\mathbf{x})^{2}+\kappa_{\bar{\gamma} \gamma}^{(j)}(\tau)\right\} \\
= & \sum_{j}\left(\tilde{\omega}_{0}^{(j)}\right)^{3}\left[\frac{\left.c\left(\tilde{\omega}_{0}^{(j)}\right)-\tilde{\omega}_{0}^{(j)} c^{\prime}\left(\tilde{\omega}_{0}^{(j)}\right)\right]}{c\left(\tilde{\omega}_{0}^{(j)}\right)^{4}}\right] \int \frac{\mathrm{d} \Omega_{\mathbf{e}}}{(2 \pi)^{2}} \\
& \times\left(\left|\Gamma^{(j)}\left(\tilde{\omega}_{0}^{(j)}, \mathbf{e}\right)\right|^{2} \chi^{(j)}(\mathbf{x})^{2}+\left|\Gamma_{\text {spon }}^{(j)}\left(\tilde{\omega}_{0}^{(j)}, \mathbf{e}\right)\right|^{2}\right)
\end{aligned}
$$


where the $\mathrm{d} \Omega_{\mathbf{e}}$ integral is taken over the unit sphere and where the contributions from $\kappa_{\bar{g} \gamma}^{(j)}$, $\kappa_{\overline{\gamma g}}^{(j)}$ have been neglected, due to (A.6). The terms have the familiar form of the Einstein coefficients in quantum optics, where there would also be a sum over polarizations. $\delta_{\text {shift }}(\mathbf{x})$ is given by

$$
\delta_{\text {shift }}(\mathbf{x})=2 \operatorname{Im} \sum_{j} \int_{0}^{\infty} \mathrm{d} \tau\left\{\kappa_{\bar{g} g}^{(j)}(\tau) \chi^{(j)}(\mathbf{x})^{2}+\kappa_{\bar{\gamma} \gamma}^{(j)}(\tau)\right\} .
$$

Since the $\kappa_{\bar{\gamma} \gamma}$ term leads to a constant it just gives an overall phase factor and can therefore be omitted.

\section{References}

[1] Szriftgiser P, Guéry-Odelin D, Arndt M and Dalibard J 1996 Phys. Rev. Lett. 774

[2] Muga J G, Sala Mayato R and Egusquiza I L (ed) 2002 Time in Quantum Mechanics (Lecture Notes in Physics vol 72) (Berlin: Springer)

[3] Schulman L S 1997 Time's Arrows and Quantum Measurement (Cambridge: Cambridge University Press)

[4] Allcock G R 1969 Ann. Phys., NY 53 253, 286, 311

[5] Kijowski J 1974 Rep. Math. Phys. 6361

[6] Giannitrapani R 1997 Int. J. Theor. Phys. 361575

[7] Aharonov Y and Bohm D 1961 Phys. Rev. 1221649

[8] Oppenheimer J, Reznik B and Unruh W G 1999 Phys. Rev. A 591804

[9] Leavens C R 2002 Phys. Lett. A 303154

[10] Egusquiza I L, Muga J G, Navarro B and Ruschhaupt A 2003 Phys. Lett. A 313498

[11] Leavens C R 2005 Phys. Lett. A 345251

[12] Halliwell J J 1999 Prog. Theor. Phys. 102707

[13] Gaveau B and Schulman L S 1990 J. Stat. Phys. 581209

[14] Schulman L S 1991 Ann. Phys., NY 212315

[15] Damborenea J A, Egusquiza I L, Hegerfeldt G C and Muga J G 2002 Phys. Rev. A 66052104

[16] Hegerfeldt G C, Seidel D and Muga J G 2003 Phys. Rev. A 68022111

[17] Navarro B, Egusquiza I L, Muga J G and Hegerfeldt G C 2003 J. Phys. B: At. Mol. Opt. Phys. 363899

[18] Damborenea J A, Egusquiza I L, Hegerfeldt G C and Muga J G 2003 J. Phys. B: At. Mol. Opt. Phys. 362657

[19] Hegerfeldt G C, Seidel D, Muga J G and Navarro B 2004 Phys. Rev. A 70012110

[20] Ruschhaupt A, Damborenea J A, Navarro B, Muga J G and Hegerfeldt G C 2004 Europhys. Lett. 671

[21] Hannstein V, Hegerfeldt G C and Muga J G 2005 J. Phys. B: At. Mol. Opt. Phys. 38409

[22] Brunetti R and Fredenhagen K 2002 Phys. Rev. A 66044101

[23] Hegerfeldt G C and Wilser T S 1992 Classical and Quantum Systems. Proc. 2nd Int. Wigner Symp. (July 1991) ed H D Doebner, W Scherer and F Schroeck (Singapore: World Scientific) p 104

Hegerfeldt G C 1993 Phys. Rev. A 47449

Hegerfeldt G C and Sondermann D 1996 Quant. Semiclass. Opt. 8121 (For a review cf [35]. The quantum jump approach is essentially equivalent to the Monte-Carlo wavefunction approach [36], and to the quantum trajectories of H Carmichael [37])

[24] Beskow A and Nilsson J 1967 Arkiv für Fysik 34561

[25] Khalfin L A 1968 Zh. Eksp. Teor. Fiz. Pis. Red. 8106 Khalfin L A 1968 JETP Lett. 865 (Engl. Transl.)

[26] Misra B and Sudarshan E C G 1977 J. Math. Phys. 18756 For further references see [38]

[27] von Neumann J 1932 Mathematische Grundlagen der Quantenmechanik (Berlin: Springer) von Neumann J 1955 Mathematical Foundations of Quantum Mechanics (Princeton: Princeton University Press) chapter 5.1 (Engl. Transl.)

[28] Lüders G 1951 Ann. Phys., Leipzig 443322

[29] Hegerfeldt G C 2003 Irreversible Quantum Dynamics (Lecture Notes in Physics vol 622) ed F Benatti and R Floreanini (Berlin: Springer) p 233

[30] Muga J G, Brouard S and Macías D 1995 Ann. Phys., NY 240351

[31] Palao J P, Muga J G and Sala R 1998 Phys. Rev. Lett. 805469

[32] Hegerfeldt G C, Neumann J T and Schulman L S 2006 Passage-time distributions from a spin-boson detector model Preprint quant-ph/0610041

[33] Peres A 1980 Am. J. Phys. 48552 
[34] Alonso D, Sala Mayato R and Muga J G 2003 Phys. Rev. A 67032105

[35] Plenio M B and Knight P L 1998 Rev. Mod. Phys. 70101

[36] Dalibard J, Castin Y and Mølmer K 1992 Phys. Rev. Lett. 68580

[37] Carmichael H 1993 An Open System Approach to Quantum Optics (Lecture Notes in Physics vol 18) (Berlin: Springer)

[38] Beige A and Hegerfeldt G C 1996 Phys. Rev. A 5353 